\title{
BMJ Open Comparative efficacy of selective serotonin reuptake inhibitors (SSRI) in treating major depressive disorder: a protocol for network meta-analysis of randomised controlled trials
}

\author{
Yongliang Jia, ${ }^{1}$ Hongmei Zhu, ${ }^{1}$ Siu-wai Leung ${ }^{1,2}$
}

To cite: Jia Y, Zhu H, Leung S-wai. Comparative efficacy of selective serotonin reuptake inhibitors (SSRI) in treating major depressive disorder: a protocol for network meta-analysis of randomised controlled trials. BMJ Open 2016;6: e010142. doi:10.1136/ bmjopen-2015-010142

- Prepublication history for this paper is available online. To view these files please visit the journal online (http://dx.doi.org/10.1136/ bmjopen-2015-010142).

Received 30 September 2015 Revised 14 December 2015 Accepted 18 January 2016

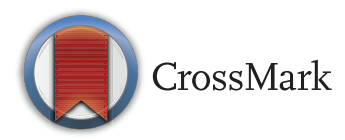

${ }^{1}$ State Key Laboratory of Quality Research in Chinese Medicine, Institute of Chinese Medical Sciences, University of Macau, Macao, China ${ }^{2}$ School of Informatics, University of Edinburgh, Edinburgh, UK

Correspondence to Dr Siu-wai Leung; siu@inf.ed.ac.uk

\begin{abstract}
Introduction: There have been inconsistent findings from randomised controlled trials (RCTs) and systematic reviews on the efficacies of selective serotonin reuptake inhibitors (SSRIs) as the first-line treatment of major depressive disorder (MDD). Besides inconsistencies among randomised controlled trials (RCTs), their risks of bias and evidence grading have seldom been evaluated in meta-analysis. This study aims to compare the efficacy of SSRIs by conducting a Bayesian network meta-analysis, which will be the most comprehensive evaluation of evidence to resolve the inconsistency among previous studies.
\end{abstract}

Methods and analyses: SSRIs including citalopram, escitalopram, fluoxetine, fluvoxamine, paroxetine, sertraline and vilazodone have been selected. Systematic database searching and screening will be conducted for the RCTs on drug treatment of patients with MDD according to pre-specified search strategies and selection criteria. PubMed, the Cochrane Library, EMBASE, ScienceDirect, the US Food and Drug Administration Website, ClinicalTrial.gov and WHO Clinical Trials will be searched. Outcome data including Hamilton Depression Rating Scale (HDRS), Montgomery-Åsberg Depression Rating Scale (MADRS) and Clinical Global Impression (CGI) from eligible RCTs will be extracted. The outcomes will be analysed as ORs and mean differences under a random-effects model. A Bayesian network metaanalysis will be conducted with WinBUGS software, to compare the efficacies of SSRIs. Subgroup and sensitivity analysis will be performed to explain the study heterogeneity and evaluate the robustness of the results. Meta-regression analysis will be conducted to determine the possible factors affecting the efficacy outcomes. The Cochrane risk of bias assessment tool will be used to assess the RCT quality, and the Grading of Recommendation, Assessment, Development and Evaluation will be used to assess the strength of evidence from the meta-analysis.

Ethics and dissemination: No ethical approval is required because this study includes neither confidential personal patient data nor interventions with patients. Protocol registration number: CRD42015024879.

\section{Strengths and limitations of this study}

- Comprehensive Bayesian network meta-analysis using both BUGS and STAN models together with sensitivity analysis, subgroup analysis and meta-regression analysis will be conducted to evaluate the efficacies of selective serotonin reuptake inhibitors (SSRIs).

- This study will provide evidence to resolve the controversy over SSRI efficacy. This study will provide the latest findings to update current clinical guidelines for treating major depressive disorder.

- This study is inherently a retrospective meta-analytic study on randomised controlled trials only.

\section{INTRODUCTION}

Major depressive disorder (MDD) is characterised by clear-cut changes in affect, cognition and neurovegetative functions and inter-episode remissions for at least 2 weeks, according to the Diagnostic and Statistical Manual of Mental Disorders Fifth Edition (DSM-5). ${ }^{1}$ Twelve-month prevalence of MDD in the USA was estimated to be about $7 \%$, with marked differences by age group such that the prevalence in 18-29-year-old individuals is threefold higher than that in individuals aged 60 years or older. ${ }^{1}$ Costs related to MDD were estimated to be US $\$ 83$ billion $^{2}$ and the projected loss of workforce productivity was up to US $\$ 24$ billion annually in the USA. ${ }^{3}$ Surveys in the UK have found the prevalence in general practitioner practice attendees ranging from $3.5 \%$ in Aberdeen $^{4}$ to $5.6 \%$ in southern England. ${ }^{5}$ According to the $\mathrm{WHO},{ }^{6} \mathrm{MDD}$ will be the largest worldwide contributor to disease burden by 2030 .

Current clinical guidelines ${ }^{7-9}$ recommended selective serotonin reuptake inhibitors (SSRI), including citalopram, escitalopram, fluoxetine, 
fluvoxamine, paroxetine, sertraline and vilazodone, as the primary pharmacotherapy of MDD, based on some findings from previous randomised controlled trials $(\text { RCT })^{10-12}$ and meta-analyses. ${ }^{13-26}$ However, the previous RCTs and meta-analyses were critically inconsistent in efficacy findings. For instance, GlaxoSmithKline's Study 329 demonstrated the antidepressant paroxetine to have remarkable efficacy and safety in treatment of MDD in adolescence ${ }^{10}$ while a re-analysis found the antidepressant paroxetine neither safe nor effective. ${ }^{13}$ An RCT found no significant difference in efficacy between sertraline and placebo groups ${ }^{11}$ but a multiple-treatment meta-analysis favoured sertraline over many antidepressants including other SSRIs. Sertraline was then deemed as the best choice for treating moderate-to-severe depression in adults. ${ }^{14} \mathrm{~A}$ randomised, double-blind, placebo-controlled trial $^{12}$ demonstrated a large and significant treatment effect, and statistically significant depressive symptom improvements among patients taken vilazodone, while a prior study ${ }^{15}$ found that the remission rates for vilazodone were not significantly different from placebo according to a systematic review on vilazodone in treating MDD. The data on clinical trials for efficacy conducted for marketing approval and submitted to the Food and Drug Administration showed that drug-placebo differences in antidepressant efficacy were small. ${ }^{16}$ Selective publication of antidepressant trials also biased a few meta-analyses for efficacy determination. ${ }^{17}{ }^{18}$ Meanwhile, the evidence based on meta-analyses was neither statistically significant nor did it favour any particular second-generation antidepressant, including SSRIs. ${ }^{19-26}$ Available evidence showed no clear differences between the efficacy of immediate-release and extended-release antidepressants including three SSRIs (fluoxetine, fluvoxamine and paroxetine) in treating MDD. ${ }^{24}$ This situation can be improved by a more methodically extensive network meta-analysis. As such, a protocol of a network meta-analysis of RCTs was published, aiming to reanalyse the efficacy, tolerability, acceptability and suicide risk of both first-generation and newer-generation antidepressants. ${ }^{25}$ However, this protocol focused on the treatment of children and adolescent depression, ${ }^{25}$ and a broader view to include adult depression would be desirable. Without proper evidence to support SSRI efficacy, a recent meta-regression study ${ }^{26}$ determined the dosedependence in treating MDD and found minimal benefits of SSRIs over placebo. Therefore, the issue about whether SSRIs are efficacious should be addressed by an improved network meta-analysis performed to rectify all known biases (table 1). The past meta-analyses performed neither sensitivity nor subgroup analyses adequately. Furthermore, only two ${ }^{19}{ }^{21}$ of the past meta-analyses evaluated the strength of evidence by the Grading of Recommendation, Assessment, Development and Evaluation (GRADE). ${ }^{27}$

\section{OBJECTIVES}

This study aims to compare the efficacy of citalopram, escitalopram, fluoxetine, fluvoxamine, paroxetine, sertraline,

Table 1 Summary of included meta-analyses

\begin{tabular}{|c|c|c|c|c|c|c|c|c|}
\hline Study & PMA & NMA & GRADE & $\begin{array}{l}\text { Subgroup and sensitivity } \\
\text { analyses }\end{array}$ & Meta-regression & $\begin{array}{l}\text { No of } \\
\text { SSRls } \\
\text { included }\end{array}$ & $\begin{array}{l}\text { Placebo } \\
\text { included }\end{array}$ & $\begin{array}{l}\text { No of } \\
\text { studies } \\
\text { included }\end{array}$ \\
\hline Ref 13 & $\sqrt{ }$ & $\sqrt{ }$ & $x$ & (1)(2) & (10) & 6 & 0 & 49 \\
\hline Ref 14 & $x$ & $x$ & $x$ & (3) & $\times$ & 1 & 1 & 8 \\
\hline Ref 15 & $\sqrt{ }$ & $x$ & $x$ & (4) & $x$ & 2 & 1 & 35 \\
\hline Ref 16 & $\sqrt{ }$ & $x$ & $x$ & (5) & $x$ & 5 & 1 & $74^{*}$ \\
\hline Ref 18 & $\sqrt{ }$ & $\sqrt{ }$ & $\sqrt{ } \dagger$ & (3)6 (7) 89 & $x$ & 6 & 1 & $>50 \ddagger$ \\
\hline Ref 20 & $\sqrt{ }$ & $\sqrt{ }$ & $\sqrt{t}$ & (3) 6)(7) (9) & $\sqrt{ }$ (no result) & 6 & 1 & $>40 \ddagger$ \\
\hline Ref 21 & $\sqrt{ }$ & $x$ & $x$ & (4) 68 & $\times$ & 6 & 1 & 21 \\
\hline Ref 22 & $\sqrt{ }$ & $x$ & $\sqrt{ }$ & (6) (7) (8) (10)(11) & $x$ & 6 & 1 & 18 \\
\hline Ref 23 & $x$ & $\sqrt{ }$ & $x$ & (17) & $x$ & 3 & 1 & 94 \\
\hline Ref 24 & $x$ & $\sqrt{ }$ & $x$ & (11)(12) & (3) (5) (7) 8)(11)(12) (10) & NA§ & Unclear & NA§ \\
\hline $\begin{array}{l}\text { Our } \\
\text { protocol }\end{array}$ & $\times$ & $\sqrt{ }$ & $\sqrt{ }$ & (1)(3)(5)(6)(7)(9)(10)(11)(12)(13)(14)(15)(16)(18) & (1)(3)(5)(7) (10)(11)(12)(13) & 7 & 1 & $\approx 1201$ \\
\hline \multicolumn{9}{|c|}{ 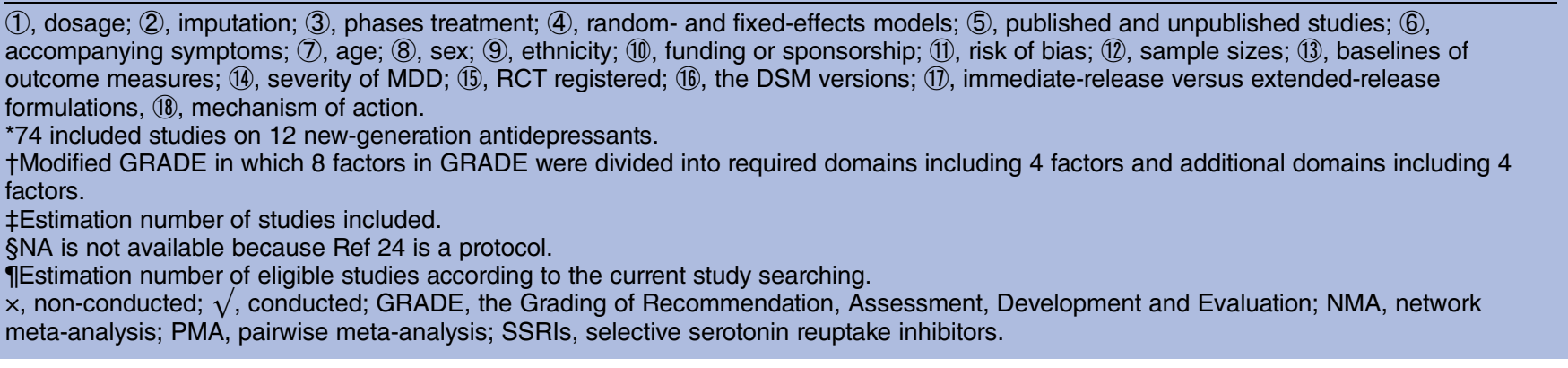 } \\
\hline
\end{tabular}


vilazodone and placebo in treating MDD, using a comprehensive Bayesian network meta-analysis on RCTs.

\section{METHODS AND ANALYSIS \\ Design}

Systematic review and Bayesian network meta-analysis.

\section{Information sources}

RCTs will be searched from PubMed, EMBASE, the Cochrane Library, ScienceDirect and PsycInfo. The following sources also will be searched for the grey literature: the US Food and Drug Administration Website, ClinicalTrial.gov and WHO Clinical Trials.

\section{Search strategies}

Drug names, major depressive disorder and 'random*' will be used as keywords to search titles or abstracts for eligible RCTs from selected databases. The search strategy was tested from March to August 2015. As an example, the following search strategy will be used for searching RCTs of citalopram for treating MDD in PubMed:

1. citalopram

2. major depressive disorder

3. random*

4. 1 in Title or Abstract

5. 2 in MeSH Terms

6. 3 in Title or Abstract

7. 4 and 5 and 6

\section{Eligibility criteria}

The retrieved reports will be screened according to the eligibility criteria shown below including participants, interventions, controls, outcome measures, types of study and other criteria.

- Participants

Inclusion: participants must be adults aged at least 18 years and suffering from MDD diagnosed using DSM criteria.

Exclusion: participants suffering from other depressive disease conditions or diagnosed using other criteria or aged 18 years or pregnant woman.

- Interventions

Inclusion: any RCT that evaluates the efficacy of selected SSRIs.

Exclusion: any RCT that evaluates other drugs or combined treatments of multiple drugs.

- Controls

Inclusion: any RCT that evaluates the efficacy of a selected drug other than the drug of intervention. Exclusion: any RCT that evaluates other drugs or combined treatments of multiple drugs.

- Outcome measures

Inclusion: efficacy outcome measures include mean difference of changes on the Hamilton Depression Rating Scale (HDRS), or Montgomery-Åsberg Depression Rating Scale (MADRS), or the Clinical Global Impression
(CGI), and treatment response in terms of the proportion of the participants who have $50 \%$ or greater improvement under the treatment, or the mean difference of scores according to HDRS or MADRS, or who receive much improved or very much improved scores in CGI. ${ }^{1}$ The primary outcome measures are HDRS or MADRS improvement and the secondary outcome measure is CGI improvement from baseline to study end. ${ }^{22} 28$

Exclusion: other outcome measures.

- Types of study

Inclusion: only RCTs will be included.

Exclusion: observational cohort and case-control studies, case reports, experimental studies and reviews will be excluded.

\section{- Other criteria}

Other inclusion criteria: the RCTs must report complete efficacy data of HDRS, MADRS or CGI of each treatment. Follow-up periods must be at least 6 weeks. Other exclusion criteria are duplicated studies or studies of combined treatments with multiple drugs.

\section{Study selection}

Reviewers will screen the retrieved database records independently according to the eligibility criteria. Disagreements between reviewers will be resolved by consensus. Selection process of studies will be shown in a PRISMA-compliant flow chart ${ }^{29}$ (figure 1).

\section{Data extraction and quality assessment}

Data of the study characteristics and the clinical outcome measures will be extracted. The data extracted from the RCTs are: (1) treatment outcome measures including HDRS, MADRS and CGI, (2) sample sizes, (3) follow-up periods, (4) dosages of interventions, (5) baselines of outcome measures, (6) phases of treatment, (7) interventions, (8) sponsorship or funding, (9) severity of MDD, (10) RCT registered, (11) accompanying symptoms, (12) the DSM versions, etc. The data will be standardised for comparability (table 2). The quality of eligible studies will be evaluated according to the Cochrane Collaboration's risk of bias tool ${ }^{30}$ (table 3).

\section{Statistical analysis}

The overall effect sizes will be determined as mean difference for continuous outcomes and ORs for dichotomous outcomes with their $95 \%$ credible intervals. Network meta-analysis based on the Bavesian hierarchical model $^{31} 32$ of included RCTs will be conducted. The differences in clinical and methodological characteristics among RCTs will be explored by subgroup analysis and sensitivity analysis. Subgroup analysis and sensitivity analysis will also be performed to evaluate the robustness of the results. Meta-regression analysis will be conducted to determine the possible factors affecting the efficacy. All drug effect parameters will be given flat normal $(0$, $0.0001)$ priors and the between-studies SD will be given flat, uniform $(0,5)$ distributions. A burn-in of 20000 simulations will be discarded. All results are based on a 
Figure 1 Process of searching and screening studies. RCT, randomised controlled trial.

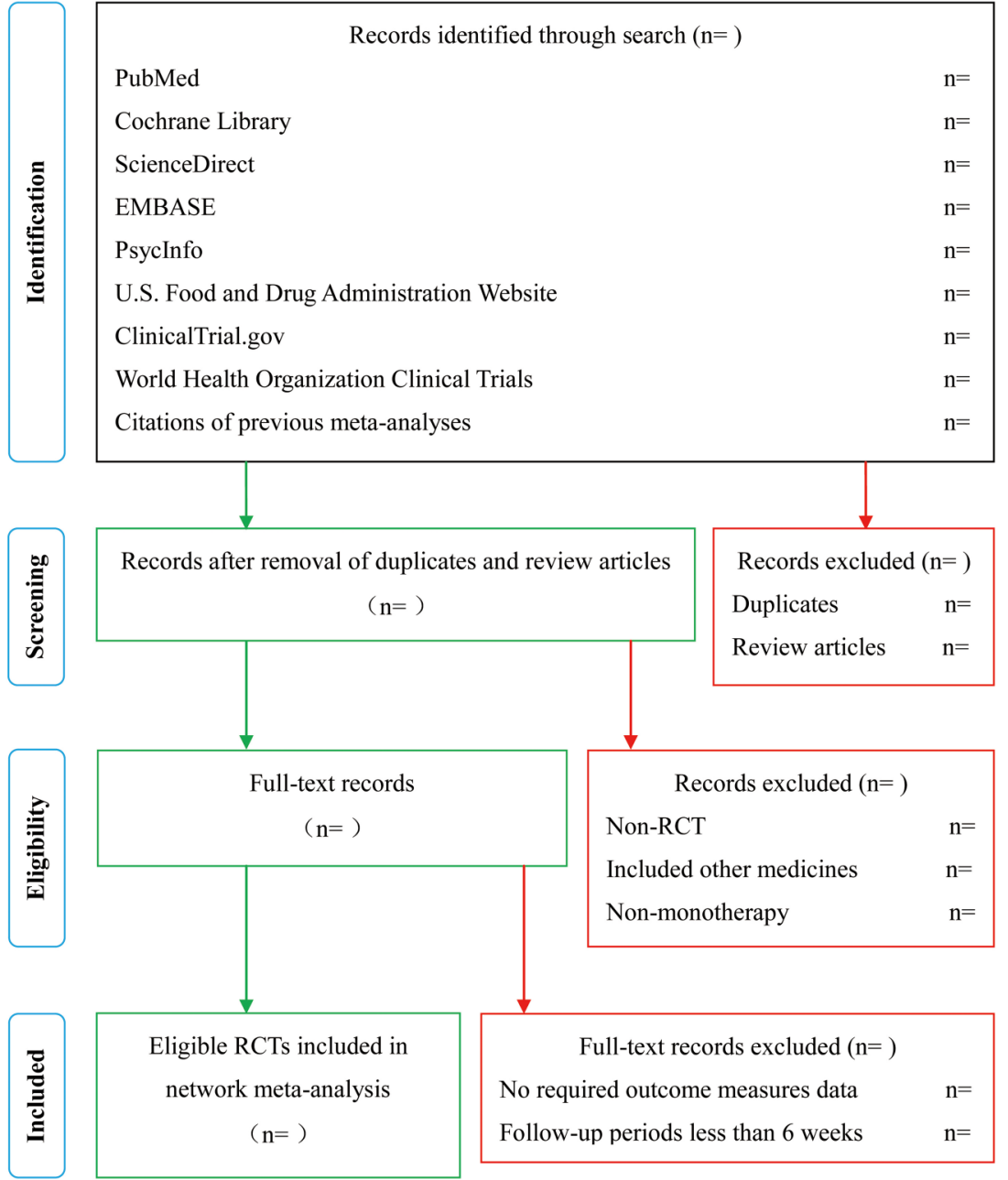

further sample of 80000 simulations. Variances and consistencies among all comparisons will be assessed using the Brooks-Gelman-Rubin method under a random-effects model. Model fitness will be assessed with the deviance information criterion (DIC) and the posterior mean of the total residual deviance. ${ }^{33}$ Bayesian inferences will be computed using a Markov-chain Monte Carlo simulation with WinBUGS, V.1.4.3 (Medical Research Council Biostatistics Unit, Cambridge, UK). ${ }^{34}$ The strength of evidence from meta-analyses for outcome measures and major comparisons will be evaluated using the GRADE approach. ${ }^{27}$ The overall ranking will be determined by the overall effect sizes. Mann-Whitney-Wilcoxon test, Kendall rank correlation and intraclass correlation coefficient will be performed with statistical software $\mathrm{R}$ ( $\mathrm{R}$ Development Core Team. R: a language and environment for statistical computing. Reference index version 2.12.0. R Foundation for Statistical Computing, Vienna, Austria. 2008) to determine the agreement among the rankings based on different outcome measures and subgroup analyses. A p value lower than 0.05 will be considered statistically significant.

\section{ETHICS AND DISSEMINATION \\ Ethical issues}

No ethical approval is required because this study includes neither confidential personal data nor interventions with patients.

Table 2 Summary of the included RCTs

\begin{tabular}{|c|c|c|c|c|c|c|c|c|c|}
\hline Study & Follow-up period & Sample size & Severity & Dosages & HDRS & MADRS & CGI & Sponsorship & $\ldots$ \\
\hline \multicolumn{10}{|l|}{ RCT 1} \\
\hline \multicolumn{10}{|l|}{ RCT 2} \\
\hline \multicolumn{10}{|l|}{ RCT 3} \\
\hline \multicolumn{10}{|l|}{ RCT 4} \\
\hline RCT 5 & & & & & & & & & \\
\hline
\end{tabular}


Table 3 RCT quality assessment according to the Cochrane Collaboration's risk of bias tool

\begin{tabular}{|c|c|c|c|c|c|c|}
\hline & $\begin{array}{l}\text { RCT } \\
1\end{array}$ & $\begin{array}{l}\mathrm{RCT} \\
2\end{array}$ & $\begin{array}{l}\text { RCT } \\
3\end{array}$ & $\begin{array}{l}\text { RCT } \\
4\end{array}$ & $\begin{array}{l}\text { RCT } \\
5\end{array}$ & $\ldots$ \\
\hline \multicolumn{7}{|l|}{$\begin{array}{l}\text { Random } \\
\text { sequence } \\
\text { generation }\end{array}$} \\
\hline \multicolumn{7}{|l|}{ Allocation } \\
\hline \multicolumn{7}{|l|}{$\begin{array}{l}\text { participants } \\
\text { and personnel }\end{array}$} \\
\hline \multicolumn{7}{|l|}{ Blinding of } \\
\hline \multicolumn{7}{|l|}{ assessment } \\
\hline \multicolumn{7}{|l|}{$\begin{array}{l}\text { Incomplete } \\
\text { outcome data }\end{array}$} \\
\hline \multicolumn{7}{|l|}{$\begin{array}{l}\text { Selective } \\
\text { reporting }\end{array}$} \\
\hline \multicolumn{7}{|l|}{ Other sources } \\
\hline
\end{tabular}

\section{Publication plan}

This protocol has been registered (registration number: CRD42015024879) with the PROSPERO (International Prospective Register of Systematic Reviews). ${ }^{35}$ The procedures of this systematic review and network meta-analysis will be conducted in accordance with the PRISMAcompliant guideline. The results of this network meta-analysis will be submitted to a peer-reviewed journal for publication.

Contributors S-wL conceived the study. YJ, HZ and S-wL wrote the protocol and approved the final manuscript.

Funding The study was supported by research grants (MYRG190-Y1-L3ICMS11-LSW and MYRG2014-00117-ICMS-QRCM) from the University of Macau.

\section{Competing interests None declared.}

Provenance and peer review Not commissioned; externally peer reviewed.

Open Access This is an Open Access article distributed in accordance with the Creative Commons Attribution Non Commercial (CC BY-NC 4.0) license, which permits others to distribute, remix, adapt, build upon this work noncommercially, and license their derivative works on different terms, provided the original work is properly cited and the use is non-commercial. See: http:// creativecommons.org/licenses/by-nc/4.0/

\section{REFERENCES}

1. American Psychiatric Association. Diagnostic and statistical manual of mental disorders. 5th edn. Washington DC: American Psychiatric Publishing, 2013:155-68.

2. Wu E, Greenberg P, Yang E, et al. Comparison of treatment persistence, hospital utilization and costs among major depressive disorder geriatric patients treated with escitalopram versus other SSRI/SNRI antidepressants. Curr Med Res Opin 2008;24:2805-13.

3. Birnbaum HG, Ben-Hamadi R, Greenberg PE, et al. Determinants of direct cost differences among US employees with major depressive disorders using antidepressants. Pharmacoeconomics 2009;27:507-17.
4. Eagles JM, Wileman SM, Cameron IM, et al. Seasonal affective disorder among primary care attenders and a community sample in Aberdeen. Br J Psychiatry 1999;175:472-5.

5. Thompson C, Thompson S, Smith R. Prevalence of seasonal affective disorder in primary care; a comparison of the seasonal health questionnaire and the seasonal pattern assessment questionnaire. J Affect Disord 2004;78:219-26.

6. World Health Organ. Global burden of disease: 2004 update. Geneva: WHO, 2004.

7. National Clinical Guidelines Centre. Depression: the Treatment and Management of Depression in Adults (Update). NICE clinical guideline 90. Leicester, UK: British Psychological Society, 2009 www.nice.org.uk/CG90.

8. American Psychiatric Association. Practice guideline for the treatment of patients with major depressive disorder 3rd edn Arlington: American Psychiatric Association, 2010:1-152.

9. Gartlehner G, Hansen RA, Morgan LC, et al. Second-generation antidepressants in the pharmacologic treatment of adult depression: an update of the 2007 comparative effectiveness review. Agency for Healthcare Research and Quality, 2011;12-EHC012-EF.

10. Keller MB, Ryan ND, Strober M, et al. Efficacy of paroxetine in the treatment of adolescent major depression: a randomized, controlled trial. J Am Acad Child Adolesc Psychiatry 2001;40:762-72.

11. Ashman TA, Cantor JB, Gordon WA, et al. A randomized controlled trial of sertraline for the treatment of depression in persons with traumatic brain injury. Arch Phys Med Rehabil 2009;90:733-40.

12. Croft HA, Pomara N, Gommoll C, et al. Efficacy and safety of vilazodone in major depressive disorder: a randomized, double-blind, placebo-controlled trial. J Clin Psychiatry 2014;75: e1291-8.

13. Le Noury J, Nardo JM, Healy D, et al. Restoring Study 329: efficacy and harms of paroxetine and imipramine in treatment of major depression in adolescence. BMJ 2015;351: h4320.

14. Cipriani A, Furukawa TA, Salanti G, et al. Comparative efficacy and acceptability of 12 new-generation antidepressants: a multiple-treatments meta-analysis. Lancet 2009;373:746-58.

15. Wang SM, Han C, Lee SJ, et al. A review of current evidence for vilazodone in major depressive disorder. Int $J$ Psychiatry Clin Pract 2013;17:160-9.

16. Kirsch I, Deacon BJ, Huedo-Medina TB, et al. Initial severity and antidepressant benefits: a meta-analysis of data submitted to the food and drug administration. PLoS Med 2008;5:e45.

17. Turner $\mathrm{EH}$, Matthews AM, Linardatos $\mathrm{E}$, et al. Selective publication of antidepressant trials and its influence on apparent efficacy. N Engl J Med 2008;358:252-60.

18. Goldacre B. Bad pharma: how drug companies mislead doctors and harm patients. Missing Data. New York: Faber and Faber, Inc, 2013:1-99.

19. Gartlehner G, Hansen RA, Morgan LC, et al. Comparative benefits and harms of second-generation antidepressants for treating major depressive disorder: an updated meta-analysis. Ann Intern Med 2011:155:772-85.

20. Fournier JC, DeRubeis RJ, Hollon SD, et al. Antidepressant drug effects and depression severity: a patient-level meta-analysis. JAMA 2010;303:47-53.

21. Gartlehner G, Gaynes BN, Hansen RA, et al. Comparative benefits and harms of second-generation antidepressants: background paper for the American College of Physicians. Ann Intern Med 2008;149:734-50.

22. Hansen RA, Gartlehner G, Lohr KN, et al. Efficacy and safety of second-generation antidepressants in the treatment of major depressive disorder. Ann Intern Med 2005;143:415-26.

23. Hetrick SE, McKenzie JE, Cox GR, et al. Newer generation antidepressants for depressive disorders in children and adolescents. Cochrane Database Syst Rev 2012;(11): CD004851.

24. Nussbaumer B, Morgan LC, Reichenpfader U, et al. Comparative efficacy and risk of harms of immediate-versus extended-release second-generation antidepressants: a systematic review with network meta-analysis. CNS Drugs 2014;28:699-712.

25. Zhou X, Qin B, Whittington C, et al. Comparative efficacy and tolerability of first-generation and newer-generation antidepressant medications for depressive disorders in children and adolescents: study protocol for a systematic review and network meta-analysis. BMJ Open 2015;5:e007768.

26. Jakubovski E, Varigonda AL, Freemantle N, et al. Systematic review and meta-analysis: dose-response relationship of selective serotonin reuptake inhibitors in major depressive disorder. Am J Psychiatry 2016;173:174-83. 
27. Atkins D, Best D, Briss PA, GRADE Working Group. Grading quality of evidence and strength of recommendations. $B M J$ 2004;328:1490-4.

28. Baer L, Blais MA. Handbook of clinical rating scales and assessment in psychiatry and mental health. Humana Press, 2010:7-35.

29. Moher D, Liberati A, Tetzlaff J, et al. Preferred reporting items for systematic reviews and meta-analyses: the PRISMA statement. PLoS Med 2009;6:1-6.

30. Higgins JP, Green S. Cochrane handbook for systematic reviews of interventions version 5.1.0 [updated March 2011]. The Cochrane Collaboration, 2011.

31. van Valkenhoef G, Lu G, de Brock B, et al. Automating network meta-analysis. Res Synth Methods 2012;3:285-99.
32. Jia Y, Leung SW. Drug efficacy in treating stable angina pectoris: a protocol for network meta-analysis of randomised controlled trials. BMJ Open 2014;4:e005453.

33. Lu G, Ades AE. Assessing evidence inconsistency in mixed treatment comparisons. J Am Stat Assoc 2006;101:447-59.

34. Lunn DJ, Thomas A, Best N, et al. WinBUGS-a Bayesian modelling framework: concepts, structure, and extensibility. Stat Comput 2000;10:325-37.

35. Jia $\mathrm{Y}, \mathrm{Zhu} \mathrm{H}$, Leung S. Comparative efficacy of selective serotonin reuptake inhibitors (SSRI) for treating major depressive disorder: a protocol for network meta-analysis of randomized controlled trials. PROSPERO 2015;CRD42015024879. http://www.crd.york.ac.uk/PROSPERO/display_record.asp? ID=CRD42015024879. 